\title{
The extreme ultraviolet and X-ray Sun in Time: High-energy evolutionary tracks of a solar-like star
}

\author{
Lin Tu${ }^{1}$, Colin P. Johnstone ${ }^{1}$, Manuel Güdel ${ }^{1}$, and Helmut Lammer ${ }^{2}$ \\ 1 Department of Astrophysics, University of Vienna, Türkenschanzstrasse 17, 1180 Vienna, Austria \\ e-mail: [lin.tu; colin.johnstone; manuel.guedel]@univie.ac.at \\ 2 Space Research Institute, Austrian Academy of Sciences, Schmiedlstrasse 6, 8042 Graz, Austria \\ e-mail: helmut.lammer@oeaw.ac.at
}

Received 20 March 2015 / Accepted 17 April 2015

\section{ABSTRACT}

\begin{abstract}
Aims. We aim to describe the pre-main-sequence and main-sequence evolution of X-ray and extreme-ultaviolet radiation of a solarmass star based on its rotational evolution starting with a realistic range of initial rotation rates.

Methods. We derive evolutionary tracks of X-ray radiation based on a rotational evolution model for solar-mass stars and the rotationactivity relation. We compare these tracks to X-ray luminosity distributions of stars in clusters with different ages.

Results. We find agreement between the evolutionary tracks derived from rotation and the X-ray luminosity distributions from observations. Depending on the initial rotation rate, a star might remain at the X-ray saturation level for very different time periods, from $\approx 10 \mathrm{Myr}$ to $\approx 300 \mathrm{Myr}$ for slow and fast rotators, respectively.

Conclusions. Rotational evolution with a spread of initial conditions leads to a particularly wide distribution of possible X-ray luminosities in the age range of 20-500 Myr, before rotational convergence and therefore X-ray luminosity convergence sets in. This age range is crucial for the evolution of young planetary atmospheres and may thus lead to very different planetary evolution histories.
\end{abstract}

Key words. Sun: evolution - stars: rotation - stars: activity - stars: solar-type - planets and satellites: atmospheres

\section{Introduction}

High-energy radiation from solar-like main-sequence (MS) stars decays over time as a result of stellar spin-down. The early Sun's X-ray $(\approx 1-100 \AA)$ and extreme-ultraviolet $(\mathrm{EUV} ; \approx 100-900 \AA)$ emissions could thus have exceeded the present-day Sun's level by orders of magnitude (Ribas et al. 2005). By driving atmospheric erosion, such extreme radiation levels were critically important for both the primordial hydrogen atmospheres (e.g. Lammer et al. 2014) and the secondary nitrogen atmospheres (Lichtenegger et al. 2010) of solar system planets. As a consequence of higher solar activity levels, stronger winds would have added to atmospheric mass loss through non-thermal processes such as ion pick-up (Kislyakova et al. 2013, 2014).

Magnetic activity is strongly linked to rotation via a stellar dynamo such that the total $\mathrm{X}$-ray luminosity decays with increasing rotation period, $P$, as $L_{\mathrm{X}} \propto P^{-3}$ to $\propto P^{-2}$; for small $P, L_{\mathrm{X}}$ saturates at $L_{\mathrm{X}} \approx 10^{-3} L_{\mathrm{bol}}\left(L_{\mathrm{bol}}\right.$ being the stellar bolometric luminosity; see Wright et al. 2011, hereafter W11). Since older (>1 Gyr) stars spin down in time approximately as $P \propto t^{0.5}$ (Skumanich 1972), $L_{X}$ decays as $L_{X} \propto t^{-1.5}$ (Güdel et al. 1997). This evolutionary trend has commonly been formulated using regression fits to $L_{\mathrm{X}}$ of stars with known ages, typically starting at the saturation level close to the zero age main sequence (ZAMS; see e.g. Güdel et al. 1997; Ribas et al. 2005).

However, stars in young stellar clusters have a wide range of rotation rates, $\Omega$, in particular at ages younger than $500 \mathrm{Myr}$ before they converge to a unique mass-dependent value (Soderblom et al. 1993). As a consequence, $L_{X}$ values also scatter over a wide range among such stars (e.g. Stauffer et al. 1994), and the age at which stars fall out of saturation depends on the star's initial $\Omega$. Given the importance of high-energy radiation in this age range for planetary atmosphere evolution, a unique description with a single radiation decay law is problematic and needs to be replaced by a description of the $L_{\mathbf{X}}$ distribution and its long-term evolution (Penz et al. 2008; Johnstone et al. 2015), spanning a wide range of possible evolutionary tracks for stars with different initial $\Omega$.

In this Letter we use a rotational evolution model to predict such luminosity distributions as a function of age, based on a range of initial $\Omega$, and we show that these predictions agree with the observed time-dependent scatter of $L_{X}$. We derive a radiative evolution model based on the full range of rotation histories for a solar-mass star, and thus find a description of the possible past histories of our own Sun, which is useful to model the corresponding evolution of solar-system planetary atmospheres. This Letter is an extension of Johnstone et al. (2015), who similarly estimated evolutionary tracks for wind properties. In this Letter, we concentrate mostly on $1 M_{\odot}$ stars, and will extend this to other stellar masses in future work.

\section{Rotation and radiation models}

As in previous studies (e.g. Gallet \& Bouvier 2013), we constrain our rotation models by assuming that the percentiles of the rotational distributions for star clusters with different ages can be combined to estimate the time evolution of a star's rotation rate. We consider only stars in the mass range $0.9 M_{\odot}$ to $1.1 M_{\odot}$. Johnstone et al. (2015) collected measured rotation periods of over 2000 stars in clusters of ages 150,550 , and $1000 \mathrm{Myr}$ 

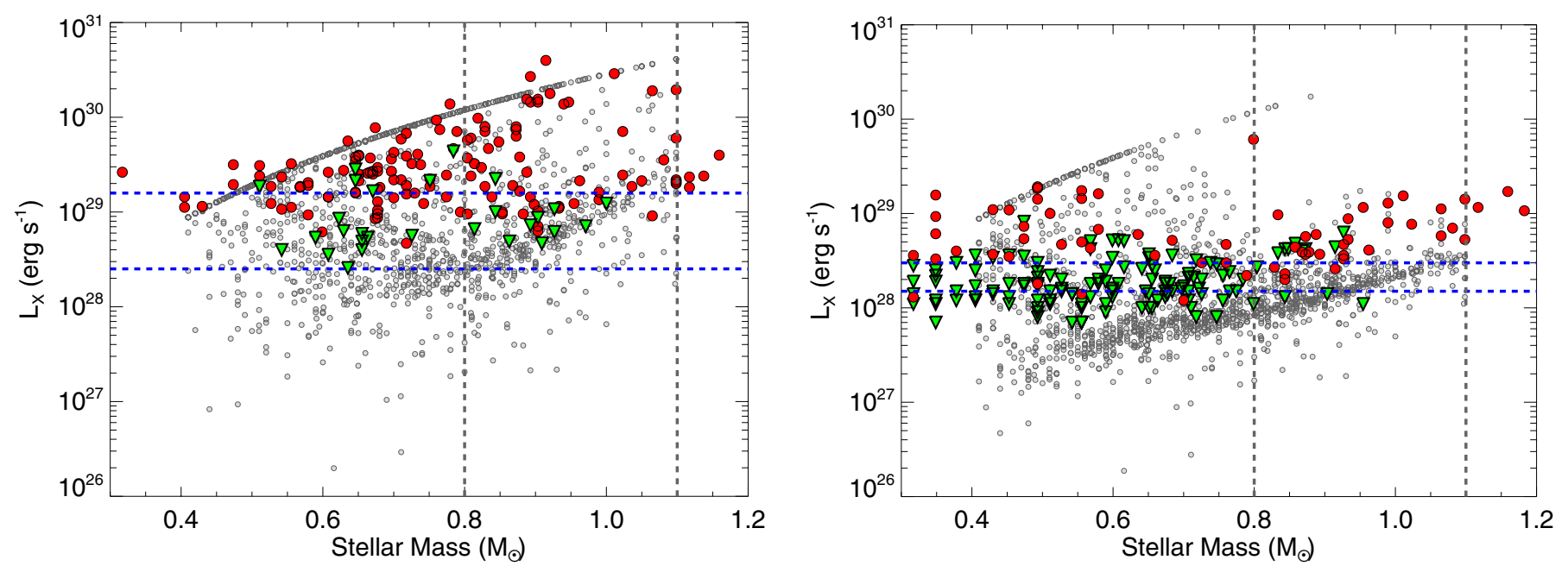

Fig. 1. Comparisons between observed and predicted distributions of $L_{\mathrm{X}}$ at ages of $150 \mathrm{Myr}(\mathrm{left})$ and $620 \mathrm{Myr}$ (right). The grey symbols show predicted distributions calculated using Eq. (1) and the distributions of rotation rates derived by Johnstone et al. (2015). The red circles and green triangles show detections and upper limits for stars in the Pleiades (left) and Hyades (right). The horizontal blue lines show detection thresholds. The upper line of stars in the theoretical distributions is caused by the stars whose rotation rates lie above the mass dependent saturation threshold.

on the MS, giving observational constraints on the percentiles at these ages (with 230, 134, and 36 stars, respectively at the considered ages). We use additional constraints for pre-mainsequence (PMS) rotation from the $\approx 2$ Myr cluster NGC 6530 (28 stars; Henderson \& Stassun 2012) and the $\approx 12$ Myr cluster h Per (117 stars; Moraux et al. 2013). For NGC 6530, the 10th, 50 th, and 90th percentiles are at $2.7 \Omega_{\odot}, 6.2 \Omega_{\odot}$, and $35.1 \Omega_{\odot}$, respectively (assuming $\Omega_{\odot}=2.9 \times 10^{-6} \mathrm{rad} \mathrm{s}^{-1}$ ), and for h Per, they are at $3.4 \Omega_{\odot}, 8.4 \Omega_{\odot}$, and $76.0 \Omega_{\odot}$, respectively.

We use an extension of the rotational evolution model of Johnstone et al. (2015). For the wind torque, we use the formula derived by Matt et al. (2012) which relates the wind torque to stellar parameters, the star's dipole field strength, $B_{\text {dip }}$, and the wind mass loss rate, $\dot{M}_{\star}$. We assume that both $B_{\text {dip }}$ and $\dot{M}_{\star}$ saturate at a Rossby number of $R o=0.13$, as suggested by the saturation of X-ray emission (W11), where $R o=P_{\text {rot }} / \tau_{\star}$ and $\tau_{\star}$ is the convective turnover time. For $\dot{M}_{\star}$, we use the scaling law derived by Johnstone et al. (2015), which is derived by fitting the rotational evolution model to observational constraints. We modify the scaling law by relating $\dot{M}_{\star}$ to $R o$; this allows us to take into account the change in $\tau_{\star}$ on the PMS. Since we only consider solar-mass stars, the scaling relation derived by Johnstone et al. (2015) can be rewritten as $\dot{M}_{\star} \propto R_{\star}^{2} R o^{-a}$. We find $a=2$ provides a good fit to the observational constraints (which is larger than the value of $a=1.33$ found by Johnstone et al. 2015). For $B_{\text {dip }}$, we use the scaling law derived by Vidotto et al. (2014) of $B_{\text {dip }} \propto R o^{-1.32}$.

To reproduce the spin-up on the PMS due to the decrease in the stellar moment of inertia, previous studies have found that core-envelope decoupling must be included (Krishnamurthi et al. 1997). We use the core-envelope decoupling model described by Gallet \& Bouvier (2015) and adopt coupling timescales of $30 \mathrm{Myr}, 20 \mathrm{Myr}$, and $10 \mathrm{Myr}$ for the 10th, 50th and 90th percentile tracks, respectively, which we find give us good agreement between the rotational evolution model and the observations. Finally, we assume that during the first few million years, the stellar rotation rates do not evolve with time due to "disklocking", i.e. magnetic interactions with the circumstellar disk. We assume disk-locking timescales of $10 \mathrm{Myr}$, $5 \mathrm{Myr}$, and $2 \mathrm{Myr}$ for the 10th, 50th, and 90th percentile tracks, respectively.
To predict $L_{\mathrm{X}}$ along the rotation tracks, we use the relation derived from MS stars by W11,

$R_{\mathrm{X}}=\left\{\begin{array}{l}C R o^{\beta}, \text { if } R o \geq R o_{\text {sat }}, \\ R_{\mathrm{X}, \mathrm{sat}}, \text { if } R o \leq R o_{\text {sat }},\end{array}\right.$

where $R o_{\text {sat }}=0.13$ is the saturation Rossby number, $R_{\mathrm{X}}=L_{\mathrm{X}} / L_{\text {bol }}$, and $R_{\mathrm{X} \text {,sat }}=10^{-3.13}$ is the saturation $R_{\mathrm{X}}$ value. We use $\beta=-2.7$ (W11). We assume that this relation can be used on the PMS if the evolution of $L_{\mathrm{bol}}$ and $\tau_{\star}$ are treated correctly. Sanz-Forcada et al. (2011) derived a power law to convert $L_{X}(5-100 \AA)$ into EUV luminosity, $L_{\mathrm{EUV}}(100-920 \AA)$, of $\log L_{\mathrm{EUV}}=4.8+0.86 \log L_{\mathrm{X}}$, where $L_{\mathrm{X}}$ and $L_{\mathrm{EUV}}$ are in erg s${ }^{-1}$.

To calculate the evolution of the stellar radius, $L_{\text {bol }}$, the moment of inertia, and $\tau_{\star}$, we use the stellar evolution models of Spada et al. (2013). However, their $\tau_{\star}$ values are approximately a factor of two above those of W11 for $1 M_{\odot}$ stars; we therefore normalise $\tau_{\star}$ at all ages such that the MS value is consistent with Eq. (1).

\section{X-ray observations}

To test our predictions for X-ray distributions at each age, we collect $L_{\mathrm{X}}$ values of single stars from ROSAT, XMMNewton, and Chandra of open clusters with ages from $30 \mathrm{Myr}$ to $620 \mathrm{Myr}$. The clusters are NGC 2547 (30 Myr; Jeffries et al. 2006), $\alpha$ Persei (50 Myr; Prosser et al. 1998), NGC 2451 (50 Myr; Hünsch et al. 2003), Blanco I (50 Myr; Pillitteri et al. 2003), Pleiades (100 Myr; Micela et al. 1999; Stauffer et al. 1994), NGC 2516 (110 Myr; Pillitteri et al. 2006; Jeffries et al. 1997), NGC 6475 (300 Myr; Prosser et al. 1995), and Hyades (620 Myr; Stern et al. 1995). For NGC 6475, since no optical catalogue was available, Prosser et al. (1995) did not report upper limits for the non-detected stars and therefore the percentiles for the distribution of $L_{X}$ should be considered upper limits. For all MS clusters, except Blanco I where masses were given, we derive masses by converting from $(B-V)_{0}$ using a relation derived from the An et al. (2007) stellar evolution models. For the PMS cluster NGC 2547, we calculated masses using the Siess et al. (2000) models. Since we use these X-ray observations only to compare them to (or: only for comparison with) 

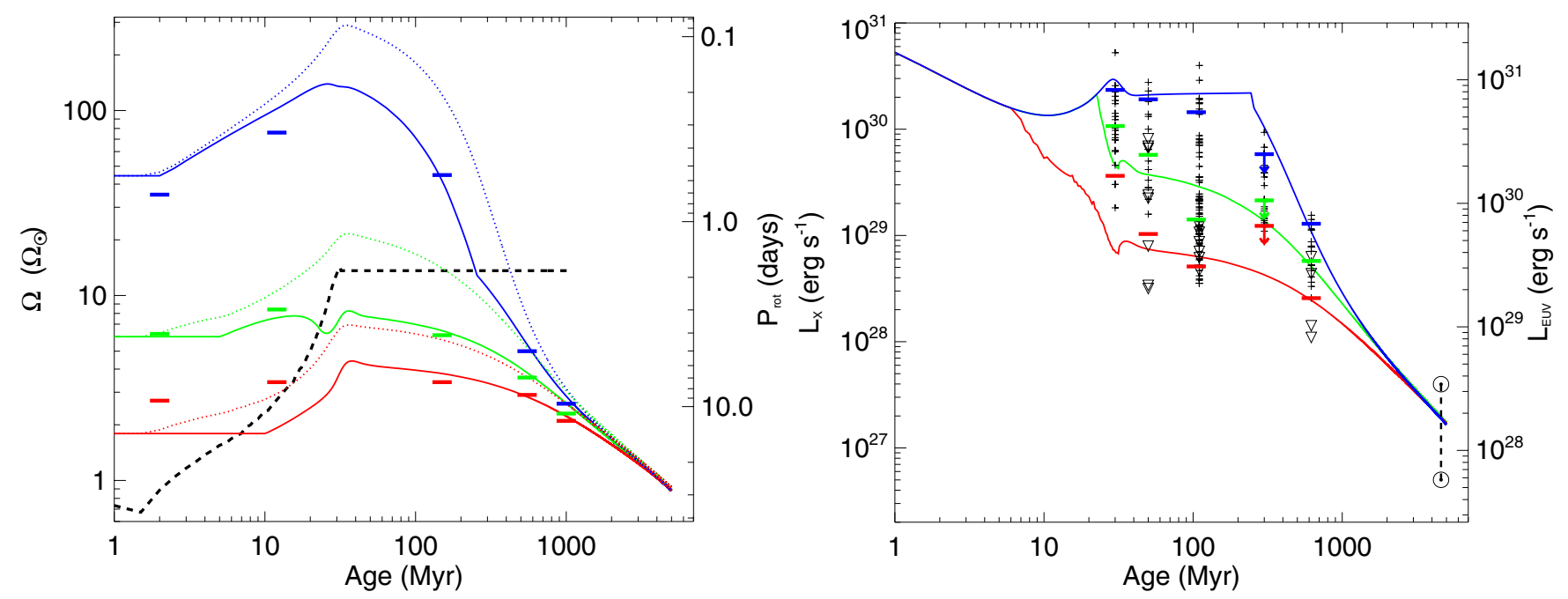

Fig. 2. Left: predicted rotational evolution tracks for stars at the 10th (red), 50th (green), and 90th (blue) percentiles of the rotational distribution. The solid and dotted lines show the envelope and core rotational evolution, respectively, and the horizontal solid lines show the observational constraints on the percentiles. The dashed black line shows the time dependent saturation threshold for $\dot{M}, B_{\text {dip }}$, and $L_{\mathrm{X}}$ calculated assuming a constant saturation $R o$ and the $\tau_{\star}$ values of Spada et al. (2013). Right: predicted $L_{\mathrm{X}}$ along each of our rotation tracks and comparisons to observed $L_{X}$ values of single stars in several clusters with upper limits shown by $\nabla$ symbols. The solid horizontal lines show the 10th, 50th, and 90th percentiles of the observed distributions of $L_{X}$ at each age calculated by counting upper limits as detections. The two solar symbols at $4.5 \mathrm{Gyr}$ show the range of $L_{\mathrm{X}}$ for the Sun over the course of the solar cycle. The scale on the right $y$-axis shows the associated $L_{\mathrm{EUV}}$.

our predictions from rotation, we do not attempt to homogenise the $M_{\star}$ and $L_{\mathrm{X}}$ determinations for each cluster. Our quantitative determinations of the $L_{X}$ tracks are based on the relation from W11 where this homogenisation was done.

\section{Results}

Johnstone et al. (2015) combined rotation period measurements of four young clusters with ages of $\sim 150 \mathrm{Myr}$ and used a rotational evolution model to predict the evolution of the resulting distribution of $\Omega$ on the MS. The sample contains 1556 stars in the $0.4-1.1 M_{\odot}$ mass range. In Fig. 1, we show predictions for the distributions of $L_{X}$ based on these $\Omega$ distributions at ages of $150 \mathrm{Myr}$ and $620 \mathrm{Myr}$ comparing them with observed values in the Pleiades and Hyades. There is good overall agreement, although intrinsic X-ray variability (typically factors of 2-3) introduces some additional scatter such as is visible for stars exceeding the saturation threshold.

To predict the range of possible $L_{\mathrm{X}}$ evolution tracks, we calculate rotation models for solar-mass stars at the 10th, 50th, and 90th percentiles of the $\Omega$ distributions, shown in Fig. 2a. Our models fit well the observational constraints on the percentiles, except for a slight underestimation of the 10th percentile track in the first $20 \mathrm{Myr}$. This might cause us to underestimate the age by a few Myr when stars on the 10th percentile track come out of saturation. Figure $2 \mathrm{~b}$ shows predicted tracks for $L_{\mathrm{X}}$ and $L_{\mathrm{EUV}}$ together with observed $L_{X}$ for stars in the 0.9-1.1 $M_{\odot}$ range for each cluster listed in Sect. 3. Because of the low number of observations in NGC 2547 (30 Myr), we extend the mass range to $0.8-1.2 M_{\odot}$. The tracks correspond very well to the observed percentiles in the individual clusters given the somewhat limited observational samples. The solar $L_{\mathrm{X}}\left(6 \times 10^{26}-5 \times 10^{27} \mathrm{erg} \mathrm{s}^{-1}\right.$, Ayres 1997; Peres et al. 2000; Judge et al. 2003) has been included as well and fits our models excellently.

Stars on our rotation tracks drop out of saturation at $\approx 6 \mathrm{Myr}$ (10th percentile, red), $\approx 20 \mathrm{Myr}$ (50th, green), and $\approx 300 \mathrm{Myr}$ (90th, blue), i.e. either as young PMS stars, as near-ZAMS stars, or as slightly evolved MS stars. The spread in $L_{X}$ amounts to as much as 1.5 orders of magnitude for several $100 \mathrm{Myr}$.

Figure 3 gives the age when a star falls out of saturation, $t_{\mathrm{sat}}$, as a function of initial $\Omega$, derived from our rotation model. This saturation time can be approximated by

$t_{\mathrm{sat}}=2.9 \Omega_{0}^{1.14}$

where $t_{\mathrm{sat}}$ is in Myr and $\Omega_{0}$ is the rotation rate at 1 Myr in units of the solar rotation rate. Assuming that the saturation level, $L_{\mathrm{X} \text {,sat }} \approx 10^{-3.13} L_{\mathrm{bol}, \odot}$, is constant in time, which is approximately true, we obtain $\log L_{X}$,sat $=30.46$. If we approximate $L_{X}$ by a power law after $t_{\text {sat }}$ (see Fig. 2b), for a given $\Omega_{0}$ we obtain

$L_{\mathrm{X}}= \begin{cases}L_{\mathrm{X}, \mathrm{sat}}, & \text { if } t \leq t_{\mathrm{sat}}, \\ a t^{b}, & \text { if } t \geq t_{\mathrm{sat}} .\end{cases}$

We require that the power law also fits the Sun with $L_{\mathrm{X}, \odot}=10^{27.2} \mathrm{erg} \mathrm{s}^{-1}$ at an age of $t_{\odot}=4570 \mathrm{Myr}$. We thus find

$b^{-1}=0.35 \log \Omega_{0}-0.98, \quad a=L_{\mathrm{X}, \odot} t_{\odot}^{-b}$.

For the 10th, 50th, and 90th percentiles in $\Omega_{0}$, corresponding to $\Omega_{0} \approx 1.8 \Omega_{\odot}, 6.2 \Omega_{\odot}$, and $45.6 \Omega_{\odot}$ with $t_{\mathrm{sat}} \approx 5.7 \mathrm{Myr}, 23 \mathrm{Myr}$, and $226 \mathrm{Myr}$, respectively, we find

$L_{\mathrm{X}}=\left\{\begin{array}{l}2.0 \times 10^{31} t^{-1.12} \\ 2.6 \times 10^{32} t^{-1.42} \\ 2.3 \times 10^{36} t^{-2.50}\end{array} L_{\mathrm{EUV}}= \begin{cases}7.4 \times 10^{31} t^{-0.96} & 10 \text { th } \\ 4.8 \times 10^{32} t^{-1.22} & 50 \text { th } \\ 1.2 \times 10^{36} t^{-2.15} & 90 \text { th }\end{cases}\right.$

where the luminosities are in $\operatorname{erg~s}^{-1}$. The slope of the median $L_{\mathrm{X}}$ track, $b=-1.42$, is very close to the values reported from linear regression to the Sun in Time sample (Güdel et al. 1997; Ribas et al. 2005). These power-law fits, valid for $t>t_{\text {sat }}$, thus describe the range of possible evolutionary tracks for $L_{\mathrm{X}}$ and $L_{\mathrm{EUV}}$. 


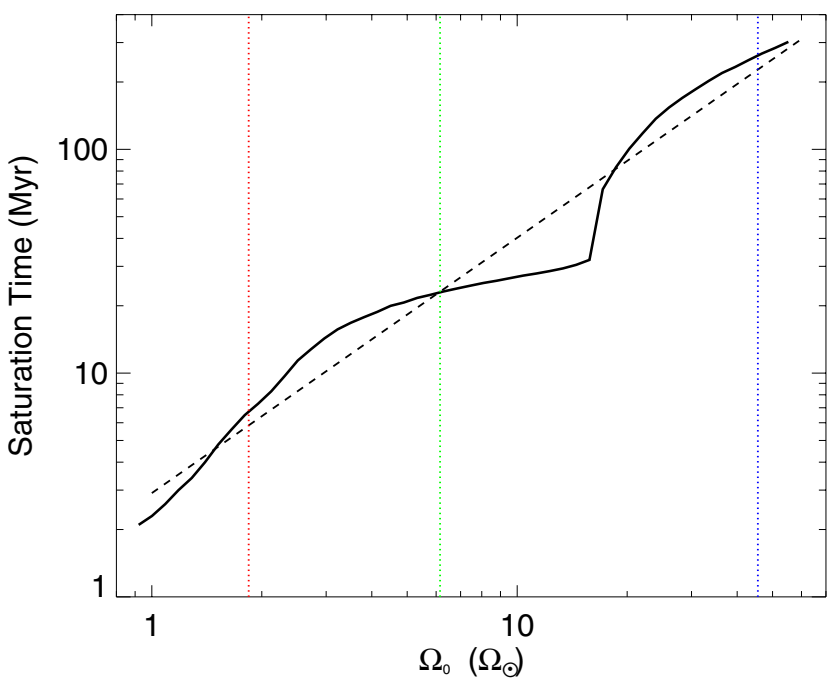

Fig. 3. Saturation time as a function of initial rotation rate $\Omega_{0}$. To calculate each rotation track, we fit power laws to the core-envelope coupling timescale and disk-locking timescales for our 10th, 50th, and 90th percentile models of $\tau_{\mathrm{CE}}=38 \Omega_{0}^{-0.34}$ and $t_{\mathrm{disk}}=13.5 \Omega_{0}^{-0.5}$, where the timescales are given in Myr and $\Omega_{0}$ is in solar units. The dashed line shows our best fit, given by Eq. (2), and the vertical lines show the saturation times of the 10th, 50th, and 90th percentile rotators.

\section{Discussion}

The large differences in the evolutionary tracks, and therefore $L_{\mathrm{X}}$ and $L_{\mathrm{EUV}}$ values, make it necessary to reconsider critically the atmospheric erosion of planets by high-energy radiation. To a first approximation, the thermal mass loss rate from a simple hydrogen dominated planetary atmosphere, $\dot{M}_{\mathrm{pl}}$, can be estimated using the energy limited approach (Watson et al. 1981; Lammer et al. 2009), where $\dot{M}_{\mathrm{pl}}$ is proportional to the incident stellar EUV flux for a given set of planetary parameters. We therefore assume that $\dot{M}_{\mathrm{pl}} \propto F_{\mathrm{EUV}}$, where $F_{\mathrm{EUV}}$ is the EUV flux at the planetary orbit. As an example, we consider the case of a $0.5 M_{\text {Earth }}$ planet at $1 \mathrm{AU}$ around a $1 M_{\odot}$ star with an initial hydrogen atmosphere of $5 \times 10^{-3} M_{\text {Earth }}$. For this case, Lammer et al. (2014) calculated $\dot{M}_{\mathrm{pl}}$ from the atmosphere of $3.5 \times 10^{32} m_{\mathrm{H} \mathrm{s}}{ }^{-1}$ with $F_{\mathrm{EUV}}=100 \mathrm{erg} \mathrm{s}^{-1} \mathrm{~cm}^{-2}$, where $m_{\mathrm{H}}$ is the mass of a hydrogen atom (see the fifth case in their Table 4). We therefore assume that $\dot{M}_{\mathrm{pl}}=5.9 \times 10^{6} F_{\mathrm{EUV}}$, where $F_{\mathrm{EUV}}$ is in erg s$~^{-1} \mathrm{~cm}^{-2}$ and $\dot{M}$ is in $\mathrm{g} \mathrm{s}^{-1}$.

We show in Fig. 4 the evolution of the planetary atmospheric mass between $10 \mathrm{Myr}$ and $5 \mathrm{Gyr}$ assuming that the central star follows the $L_{\mathrm{EUV}}$ tracks shown in Fig. 2. In all three cases the planet at $10 \mathrm{Myr}$ has identical atmospheric masses; however, by $5 \mathrm{Gyr}$ the atmospheric hydrogen contents are very different. Orbiting the slowly rotating star, the planet retains $45 \%$ of its initial atmosphere; orbiting the rapidly rotating star, the planet loses its entire atmosphere within $100 \mathrm{Myr}$; orbiting the 50th percentile rotator, the planet also loses its atmosphere, but this instead takes almost a Gyr. Although this is a simple calculation of a single example atmosphere, it is sufficient to show that the star's initial rotation rate - and the subsequent rotational evolution - is an important aspect that needs to be properly considered when studying the evolution of the atmospheres of terrestrial planets.

Acknowledgements. The authors thank the referee, Nicholas Wright, for valuable comments. L.T. was supported by an "Emerging Fields" grant of the University of Vienna through the Faculty of Earth Sciences, Geography and Astronomy. C.P.J., M.G., and H.L. acknowledge the support of the FWF NFN project S11601-N16 "Pathways to Habitability: From Disks to Active

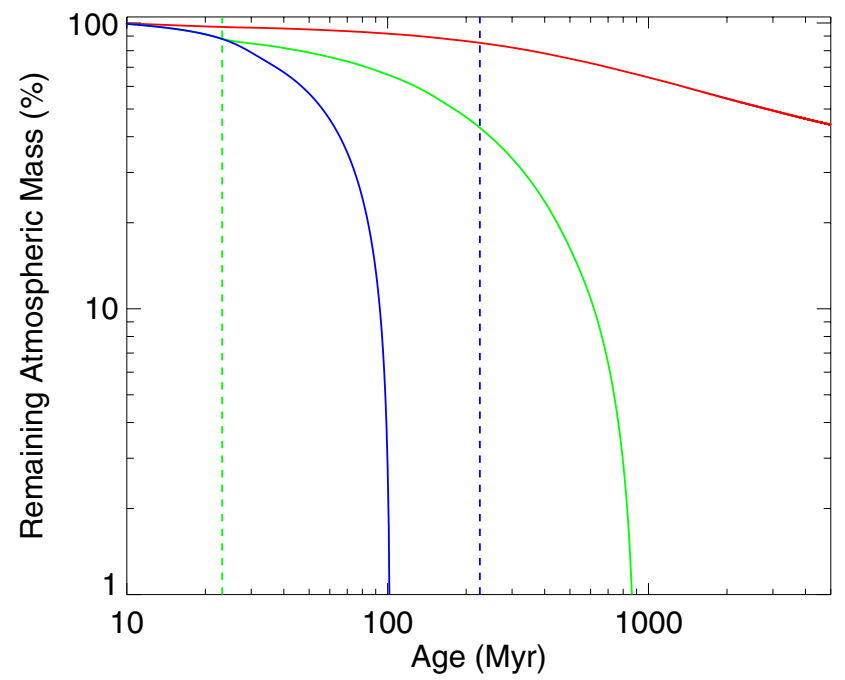

Fig. 4. Evolution of the planetary atmospheric mass of a $0.5 M_{\text {Earth }}$ planet orbiting a $1 M_{\odot}$ star at 1 AU with an initial mass of $5 \times 10^{-3} M_{\text {Earth }}$. The tracks correspond to planets orbiting stars that are in the 10th (red), 50th (green), and 90th (blue) percentiles of the rotational distributions. The vertical lines show the stellar saturation times.

Stars, Planets and Life", and the related FWF NFN subprojects S11604-N16 "Radiation \& Wind Evolution from T Tauri Phase to ZAMS and Beyond" and S11607-N16 "Particle/Radiative Interactions with Upper Atmospheres of Planetary Bodies Under Extreme Stellar Conditions". This publication is supported by the Austrian Science Fund (FWF).

\section{References}

An, D., Terndrup, D. M., \& Pinsonneault, M. H. 2007, ApJ, 671, 1640 Ayres, T. R. 1997, J. Geophys. Res., 102, 1641

Gallet, F., \& Bouvier, J. 2013, A\&A, 556, A36

Gallet, F., \& Bouvier, J. 2015, A\&A, 577, A98

Güdel, M., Guinan, E. F., \& Skinner, S. L. 1997, ApJ, 483, 947

Henderson, C. B., \& Stassun, K. G. 2012, ApJ, 747, 51

Hünsch, M., Weidner, C., \& Schmitt, J. H. M. M. 2003, A\&A, 402, 571

Jeffries, R. D., Thurston, M. R., \& Pye, J. P. 1997, MNRAS, 287, 350

Jeffries, R. D., Evans, P. A., Pye, J. P., \& Briggs, K. R. 2006, MNRAS, 367, 781

Johnstone, C. P., Güdel, M., Brott, I., \& Lüftinger, T. 2015, A\&A, 577, A28

Judge, P. G., Solomon, S. C., \& Ayres, T. R. 2003, ApJ, 593, 534

Kislyakova, K., Lammer, H., Holmström, M., et al. 2013, Astrobiology, 13, 1030 Kislyakova, K. G., Johnstone, C. P., Odert, P., et al. 2014, A\&A, 562, A116

Krishnamurthi, A., Pinsonneault, M. H., Barnes, S., \& Sofia, S. 1997, ApJ, 480, 303

Lammer, H., Odert, P., Leitzinger, M., et al. 2009, A\&A, 506, 399

Lammer, H., Stökl, A., Erkaev, N. V., et al. 2014, MNRAS, 439, 3225

Lichtenegger, H., Lammer, H., Grießmeier, J., et al. 2010, Icarus, 210, 1

Matt, S. P., MacGregor, K. B., Pinsonneault, M. H., \& Greene, T. P. 2012, ApJ, 754, L26

Micela, G., Sciortino, S., Harnden, Jr., F. R., et al. 1999, A\&A, 341, 751

Moraux, E., Artemenko, S., Bouvier, J., et al. 2013, A\&A, 560, A13

Penz, T., Micela, G., \& Lammer, H. 2008, A\&A, 477, 309

Peres, G., Orlando, S., Reale, F., Rosner, R., \& Hudson, H. 2000, ApJ, 528, 537

Pillitteri, I., Micela, G., Sciortino, S., \& Favata, F. 2003, A\&A, 399, 919

Pillitteri, I., Micela, G., Damiani, F., \& Sciortino, S. 2006, A\&A, 450, 993

Prosser, C. F., Stauffer, J. R., Caillault, J.-P., et al. 1995, AJ, 110, 1229

Prosser, C. P., Randich, S., \& Simon, T. 1998, Astron. Nachr., 319, 215

Ribas, I., Guinan, E. F., Güdel, M., \& Audard, M. 2005, ApJ, 622, 680

Sanz-Forcada, J., Micela, G., Ribas, I., et al. 2011, A\&A, 532, A6

Siess, L., Dufour, E., \& Forestini, M. 2000, A\&A, 358, 593

Skumanich, A. 1972, ApJ, 171, 565

Soderblom, D., Stauffer, J., MacGregor, K., \& Jones, B. 1993, ApJ, 409, 624

Spada, F., Demarque, P., Kim, Y.-C., \& Sills, A. 2013, ApJ, 776, 87

Stauffer, J. R., Caillault, J.-P., Gagne, M., Prosser, C. F., \& Hartmann, L. W. 1994, ApJS, 91, 625

Stern, R. A., Schmitt, J. H. M. M., \& Kahabka, P. T. 1995, ApJ, 448, 683 Vidotto, A. A., Gregory, S. G., Jardine, M., et al. 2014, MNRAS, 441, 2361

Watson, A. J., Donahue, T. M., \& Walker, J. C. G. 1981, Icarus, 48, 150

Wright, N., Drake, J., Mamajek, E., \& Henry, G. 2011, ApJ, 743, 48 (W11) 